\title{
FAITS, FICTIONS, FUMÉES
}

Pour compléter l'allitération, j'étais prêt à ajouter un quatrième substantif, plus nauséabond et brutal, mais la revue risquait une assignation en justice pour diffamation envers un chef d'État. Donc rabattons-nous sur les faits - tout en sachant que la frontière entre fictions et fumées est encore plus poreuse que la séparation entre faits et fictions - et commençons par les inventaires ne prêtant pas à discussion.

Le travail de Paulus Gerdes ${ }^{1}$ est un instrument indispensable aux mozambicanistes - et a fortiori aux Mozambicains envisageant d'effectuer des recherches approfondies. Il recense 314 thèses soutenues par leurs compatriotes dans le monde entier, et 319 autres présentées sur le Mozambique par des étrangers, depuis la première, celle d'Eric V. Axelson en 1938. C'est dire que l'irruption du pays dans les études universitaires est un phénomène notable, mais récent. Son texte est une compilation de diverses sources, sans qu'elles aient fait l'objet d'une critique ou de rectifications de la part de Gerdes. Les erreurs sont cependant rares (ex.: l'université de Haute-Bretagne n'est pas située à Villeneuve-d'Ascq et si l'île Europa est bien dans le canal de Mozambique, elle n'a rien à voir avec son homonyme sur le continent). Il a eu l'excellente idée de ventiler les thèses par pays de soutenance et par nationalité des candidats. On apprend ainsi que les Portugais n'ont soutenu que 37 thèses mozambicanistes au Portugal et les Mozambicains que 22 dans les universités portugaises. En revanche, les Mozambicains en ont présenté 43 en Allemagne et les Allemands probablement plus de 45 dans leur pays même. Il s'agit évidemment de s'entendre sur ce qu'on appelle une thèse de doctorat et, personnellement, j'ai plus confiance en la qualité d'une thèse au Portugal qu'en celle d'un travail bâclé et soutenu grâce à la complaisance d'un jury en Allemagne de l'Est ou au Brésil. Le lieu de soutenance dépend également des bourses ou des facilités offertes par le pays d'accueil. Que peut bien trouver sur place, en matière de sources mozambicanistes, un candidat mozambicain en Espagne (13), en Suède (19), en Russie (6), pour ne rien dire de pays tels que l'Italie (14) ou d'autres qui se sont mis à débiter des thèses à la pression comme dans certaines disciplines en France, voire en d'« obscures » contrées telles que l'Australie (5) ou l'Ukraine (3) ? Derrière les faits, bruts de décoffrage, que livre le répertoire, on pourrait donc élaborer une analyse sur la politique culturelle des pays «donateurs ». On n'a pas le temps et c'est bien dommage.

Inventário de Timor ${ }^{2}$ est la simple énumération et description technique de pièces d'archives émanant de trois Directions (Travaux publics et Communications; Mines; Économie) et du Conseil supérieur de la mise en valeur de l'Outre-Mer

${ }^{1}$ P. Gerdes, Doctoral Theses by Mozambicans and About Mozambique, Durban, Lulu Press/Just Done Productions Publishing et Nampula, Universidade Lúrio, 2007, 123 p., ISBN : 978-1-43031898-9.

${ }^{2}$ Collectif, Inventário de Timor, Lisbonne, Instituto Português de Apoio ao Desenvolvimento, 2003, 199 p., ISBN : 972-96666-9-5. 
appartenant à l'ancien ministère de l'Outre-Mer, telles qu'elles ont été recueillies par l'Instituto Português de Apoio ao Desenvolvimento. Le nombre de pages n'est pas indiqué. À noter la richesse des rapports sur le pétrole et sa prospection. Preuve que les fonctionnaires avaient recueilli une masse de données (plus de 200 pièces), probablement largement inexploitées par leurs successeurs actuels.

Avec Les économies de l'Afrique centrale $2006^{3}$, nous avons quitté les seuls faits bruts pour entrer dans des appréciations pas toujours édulcorées sur la situation économique et les modalités appliquées par les bureaucrates locaux pour développer leur pays. Ce rapport officiel concerne onze États, dont l'Angola, São Tomé e Príncipe et la Guinée équatoriale. C'est souvent accablant pour les administrations luso-africaines (ex. : pour créer une entreprise en Angola, il faut franchir quatorze étapes étalées sur une période moyenne de 146 jours, contre douze étapes et 37 jours au Cameroun). Mais ne retenons que les statistiques fournies par les auteurs africains du rapport qui sont, évidemment, des plus utiles pour les économistes... et les investisseurs potentiels s'ils ne se découragent pas facilement.

Beaucoup plus encyclopédique, l'ouvrage ${ }^{4}$ d'un professeur de génie mécanique à l'université Eduardo Mondlane en 2005 semble s'adresser aux voyageurs et aux membres d'ONG travaillant sur place. Après un petit résumé historique, il décrit la situation sociopolitique, la santé, les religions, les rapports interethniques, l'information, la culture, l'enseignement, l'économie, les possibilités touristiques, l'aide extérieure, etc. Il offre des renseignements peu fréquents sur les activités des centres culturels étrangers et ne s'interdit pas des coups de patte (ex: la fausse solidarité de façade de la population de l'ex-République démocratique allemande). Retour sur l'Angola avec un livre que l'on ne trouvera pas facilement dans les bibliothèques situées hors d'Angola, la politique des éditeurs locaux en matière de diffusion étant, pour le moins, difficile à comprendre. Si l'auteur ne se décide pas à faire lui-même son service de presse, il peut être assuré que rarissimes seront ses lecteurs étrangers, tant le mot «exporter» est un vocable quasiment inconnu, voire incongru, pour les éditeurs locaux. C'est la bonne vieille tradition héritée de Cuba: seul le marché intérieur intéresse chaque Palop se piquant de culture livresque.

Alors combien seront les lecteurs de la thèse ${ }^{5}$ de José Gonçalves (alias Jonuel Gonçalves) qui se présente comme économiste passionné par la province du Cunené. Moi aussi, je suis passionné par cette région, mais pas pour les mêmes raisons puisqu'elles sont purement historiographiques. Et comme je n'ai aucune compétence en hydraulique, je me bornerai à ajouter que, selon l'auteur, le barrage de Calueque a été bombardé, mais sans que cela nuise à sa capacité de rétention, et que les Namibiens continuent à pomper l'eau vers leur territoire sans rien verser à l'Angola $^{6}$, à la date de rédaction. Latent, le problème de l'utilisation des eaux du

${ }^{3}$ Collectif (Commission économique des Nations unies pour l'Afrique. Bureau Afrique centrale), Les économies de l'Afrique centrale 2006, Paris, Maisonneuve \& Larose, 2006, 251 p., ISBN : 2-7068-1982-0 et 2-86877-214-5.

${ }^{4}$ R. Steinbuch, Mosambik. Schwarz und arm und ziemlich weit weg, Stuttgart, Schmetterling Verlag, 2007, 207 p., photos noir et blanc, ISBN : 3-89657-011-0.

${ }^{5} \mathrm{~J}$. Gonçalves, Valoração da água em economia de desenvolvimento. Estudo com base em trechos dos rios Cunene e Uruguai, Luanda, Editorial Nzila, 2005, 151 p., ISBN : 972-8823-84-3.

${ }^{6}$ Ibid. : 68. 
Cunené ne peut donc que se compliquer avec le projet de barrage sur le cours mitoyen, à Epupa. Ce texte va loin dans son étude chiffrée des incidences de cette captation des eaux du fleuve angolais sur l'élevage et l'agriculture des deux côtés de la frontière.

Un bref coup de chapeau au Tchèque Jan Klíma qui poursuit son travail de pionnier des études lusophones (histoire et politique) dans son pays avec, lui aussi, une présentation encyclopédique de la Guinée-Bissau ${ }^{7}$ à l'usage de ses compatriotes. J'admets que l'on éprouve une douce euphorie à être le premier dans sa langue à parler d'un pays inconnu. Il ne prétend pas apporter d'éléments nouveaux puisqu'il résume ce qu'on trouve dans les livres étrangers, notoirement absents des bibliothèques locales. Que Prague ait d'autres priorités que l'acquisition de livres en anglais, portugais, français, etc., sur les Palop ne surprendra personne. Après tout, combien de livres en tchèque trouve-t-on dans les universités portugaises, et même à São Paulo ou à Rio?

Pourtant, plus ou moins artificiels et soumis aux aléas de financement officiels ou privés, on voit arriver les actes bilingues d'un colloque international consacré à la lusophonie africaine en 2005 et réunissant à Prague des spécialistes (ou des étudiants) tchèques, angolais et portugais. Défalcation faite des prestations cosmétiques de quelques habitués de ces circuits plus mondains et politiques que véritablement novateurs, on citera un texte ${ }^{8}$ sur les relations entre la Guinée-Bissau (et le PAIGG) et la Tchéquie entre 1961 et 1989, notamment dans le domaine des services secrets et de la police. Il en reste toujours quelques bonnes habitudes, puisées auprès de maîtres diligents.

Et puisque nous sommes dans les livres à diffusion problématique hors de leur lieu d'édition, enchaînons sur un texte publié au Sénégal, dans une organisation internationale où un vulgaire bibliographe africaniste a peu de chances de voir ses demandes réitérées d'un modeste service de presse obtenir une quelconque réponse de la part des puissants personnages qui dirigent ces olympiennes instances. Le livre ${ }^{9}$ en question est pourtant le premier à avoir été publié en portugais sur les politiques, l'économie et les sociétés palopiennes* par le Codesria (Conseil pour le développement de la recherche en sciences sociales en Afrique), actif - en principe - depuis 1973. Ne nous laissons donc pas désarçonner par ce mutisme bureaucratique et signalons que "Lusofonia" em África n'est pas pire et est même meilleure que ces habituelles juxtapositions de contributions qui se publient torrentiellement chez les anglophones et, par imitation compulsive sinon maladive, chez les autres locuteurs de «langues mineures» ayant des spécialistes à mettre en valeur ou à faire se rencontrer. Cela donne du travail aux hôteliers et c'est souvent inspiré de bonnes intentions. Pour ces ouvrages collectifs l'argent manque rarement, contrairement à l'originalité des textes qu'ils insèrent. Sur le plan concret,

${ }^{7}$ J. KLíma, Guinea-Bissau, Prague, Nakladatelství Libri, 2006, 138 p., ISBN : 80-7277-329-1.

${ }^{8}$ M. Caetano, P. Jelínek \& A. Knitl (eds), Lusofonni Afrika 1975-2005 - África lusófona 19752005, Prague, s.e., 2006, 207 p., ISBN : 80-86506-49-5.

${ }^{9}$ T. Cruz e Silva, M.G. Mendes de Araújo \& C. Cardoso (eds), "Lusofonia em África». História, democracia e integração africana, Dakar, CODESRIA, 2005, xii-248 p., ISBN : 2-86978-174-1.

* Ndlr: "sociétés palopiennes", c'est-à-dire relatives aux Palop (Pays africains de langue officielle portugaise). 
au-delà des généralités, on trouvera dans ces actes de textes en portugais des études sur les institutions universitaires et la recherche au Mozambique, les familles des bidonvilles de Luanda et de Maputo, la construction nationale en Guinée-Bissau et au Cap-Vert, la diversité linguistique en Angola, l'État-nation et le rôle de la chefferie en Guinée, etc. Les sociologues, politologues et anthropologues tiennent le haut du pavé dans ce recueil et montrent une vitalité qui n’a rien à envier à celle de leurs confrères plus connus parce que publiant en anglais, voire en français. Donc, tout est pour le mieux et autant le faire savoir hors du ghetto.

Le Camerounais qui a soutenu une thèse ${ }^{10}$ en sciences politiques à l'université de Kassel ne doute de rien puisqu'il mobilise les principaux facteurs entravant l'émergence d'un État viable en Afrique noire en examinant l'héritage de quatre colonisations dont la portugaise en Angola. À partir d'une bibliographie très lacunaire, il se condamne donc à picorer chez ses devanciers ayant traité avec plus ou moins de détails l'école coloniale, le principe de territorialité, le pacte colonial, l'économie, etc. Pour l'Angola, il est parfois disert sur des problèmes non essentiels et trop rapide sur d'autres (ex: le vertige du pouvoir, la corruption). Festival d'arguments à l'intention des professeurs de sciences politiques, qui va les lire à Luanda? Dans la vraie vie, c'est-à-dire loin des universitaires européens, des occupations plus rentables requièrent toute l'attention des ministres et des généraux. Mais félicitons l'auteur d'avoir déjà consulté quarante livres ou articles sur l'Angola, alors que ses confrères locaux pourraient difficilement trouver un cinquième de sa liste dans les bibliothèques de Lubango ou de Luanda. Est-on dans les fictions ou les fumées?

Circonscrite dans le temps, mais non dans l'espace, la thèse de science politique de Béatrice Pouligny aura des retombées plus aisément perceptibles puisque l'auteure a travaillé pour l'ONU et des ONG s'intéressant, à divers titres, au rétablissement de la paix, tel qu'il est mis en œuvre par les militaires et les autres envoyés dépêchés par le Conseil de sécurité. L'originalité - et elle est grande - de son travail provient du fait qu'elle se place du côté des populations, des «gens d'en bas » confrontés à ces bons Samaritains censés les protéger. Elle montre ce qui devrait être une évidence - même pour les diplomates et fonctionnaires onusiens -, à savoir que le succès de ces coûteuses opérations dépend avant tout de la façon dont les «sauveurs » sont perçus par les « sauvés », c'est-à-dire du contexte politique, économique, social, ethnoreligieux, mental, local, etc. En d'autres termes, l'auteure pointe du doigt ce qui ne va pas dans ces confrontations de mentalités et fournit de nombreux exemples qu'elle a relevés personnellement au Cambodge, en Somalie, en Bosnie, en Haiti, en Sierra Leone et au Mozambique. Quinze ans de terrain et d'enquêtes l'habilitent largement à indiquer ce qu'il faut faire ou ne pas faire. L'architecture de son livre est thématique et non pas chronologique ou géographique, mais un index fort bien fait permet de voir ce qui appartient au Mozambique. J'y apprends, notamment, pourquoi le ministre de la Défense du Frelimo (Front

${ }^{10}$ M.L. Jamfa Chindjeu, Comment comprendre la "crise" de l'État postcolonial en Afrique? Un essai d'explication structurelle à partir des cas de l'Angola, du Congo-Brazzaville, du Congo-Kinshasa, du Libéria et du Rwanda, Berne, Peter Lang, 2005, xii-460 p., ISBN : 3-03910-689-9. 
de libération du Mozambique) freinait des quatre fers pour entraver l'application des accords de paix : les trafics qu'il contrôlait pendant le conflit ne lui avaient pas encore rapporté assez d'argent pour qu'il puisse prendre une retraite dorée comme les autres généraux, anciens héros de la guerre de libération, recasés dans les « affaires ». Dans l'autre camp, Dhlakama, chef de la Renamo (Résistance nationale mozambicaine), traînait les pieds afin d'obtenir toujours plus d'argent pour lui, son état-major et ses affidés. 15 millions de dollars fournis par l'ONU ne suffisaient pas à satisfaire ces défenseurs de la liberté d'entreprise. Bref, dans ce livre ${ }^{11}$ réaliste, c'est le dessous des cartes diplomatiques qui nous est décrit. Des tonnes de détergent n'arriveraient pas à les rendre propres. On était loin de 1964 et encore plus du «massacre de Mueda». En plein dans le "para sueco ver», selon la formule consacrée à Maputo.

Et puisqu'on est dans les textes à propos desquels on a envie de dire du bien, allons voir du côté de Maître Renard finassant avec les prélats. On n'est pas chez La Fontaine, mais dans une excellente tese de mestrado ${ }^{12}$ qui pourrait en remontrer à bien des doctorats obtenus à la sauvette. Salazar e o Vaticano détruit plusieurs vérités longtemps tenues pour intouchables, la première étant que, catholique, le vieux goupil était de mèche avec la Papauté pour perpétuer le colonialisme au mieux des intérêts des deux parties. À l'issue d'une analyse très poussée de ce que l'on donne en consultation dans les archives de Lisbonne, l'auteur conclut au contraire que Salazar a pratiquement sacrifié ses convictions religieuses lorsqu'elles se heurtaient à sa politique nationaliste, c'est-à-dire coloniale, voire impériale. On ne peut pas détailler ici la serpentine politique du grand reclus, mais qui veut connaître les méandres diplomatiques du Concordat, de l'Accord missionnaire, des crises des années cinquante en Orient et en Afrique, de l'antagonisme diffus ou affiché entre sa conception de l'Ultramar et les objectifs poursuivis par l'Église en matière de décolonisation, etc., doit considérer ce livre comme son Évangile provisoire, si l'on peut dire. On est loin des fumées d'encens, mais pas encore dans la fiction.

Celle-ci arrive sans fard et sans masque avec The Mozambique Affair ${ }^{13}$, le roman d'un Rhodésien - ils ne meurent jamais - qui, dans sa jeunesse, a eu des relations étroites avec deux microsociétés imbriquées au Mozambique colonial: le petit monde des chasseurs d'ivoire, d'extraction britannique, et celui, plus étendu, des planteurs portugais au Manica (avec des antennes dans la Gorongosa et à Beira). Le meilleur est au début: le retour d'un vieux Nemrod en 1981 qui veut retrouver sa maison dont il a été chassé en 1975 par le Frelimo. Qui connaît L'okapi de Philippe Diolé dont le héros traversait l'Angola pour remonter dans un Congo apocalyptique en 1961 ne pourra qu'être sensible à cette atmosphère de Darkest Africa postcoloniale. On laissera le lecteur découvrir cette ambiance d'abattoir.

11 B. Pouligny, Ils nous avaient promis la paix. Opérations de l'ONU et populations locales, Paris, Presses de la Fondation nationale des sciences politiques, 2004, 356 p., ISBN : 2-7246-0947-6.

${ }^{12}$ B.C. Reis, Salazar e o Vaticano, Lisbonne, Imprensa de Ciências Sociais, 2006, 351 p., ISBN : 972-671-183-5.

${ }^{13}$ H. Wessels, The Mozambique Affair, Durban, Just Done Productions Publishing, 2006, 168 p., ISBN : 978-1-9201-6906-0. 
Signalons, cependant, quelques épisodes mozambicains déjà rencontrés dans la littérature des Rhodésiens en exil : la chute du moral des troupes portugaises à Beira et à Tete (parachutistes inclus); le massacre de Wiriyamu; la capture des survivants d'une tuerie de planteurs par le Frelimo qui les détiendra dans un camp de prisonniers. Wessels va plus loin dans ce registre macabre, car il décrit en détail le viol public d'une jeune Portugaise par le «monstre suprême », selon lui : le chef guérillero du Frelimo, Sebastião Mabote qui en fera - invraisemblablement - son esclave sexuelle pendant deux ans, jusqu'à ce qu'elle soit délivrée par une attaque des Rhodésiens qui tuent ce Mabote fictif (vers 1976-1977). Sauf erreur de ma part, ce Mabote, dans la vie réelle, avait survécu à la guerre civile puisqu'il périt noyé sur une plage du Sud-Mozambique en 2001 ! Et l'affaire se termine plutôt bien : les Rhodésiens réconciliés avec les Mozambicains doivent venir cultiver les plantations abandonnées par les Portugais. Embrassons-nous entre Africains.

Un livre $^{14}$ rare et franchement exotique ? Celui d'un médecin nicaraguayen qui, par petites touches, raconte ses onze ans d'expériences au Mozambique de 1990 à 2001. D'abord à Maputo dans une ONG italienne, cet humaniste en blouse blanche organise une coopérative pour les travailleurs mozambicains expulsés d'Allemagne et grugés par les fonctionnaires du Ministère. Devenu épidémiologiste, il assiste en 1994 au retour des réfugiés au Malawi qui apportent la peste bubonique, et il parcourt ensuite la Zambézia et le Niassa pour lutter contre toutes sortes d'épidémies (600 morts de méningite au Cabo Delgado). Malgré son statut social, il est proche des ruraux (une scène de confraternisation alcoolique chez les Chope de l'Inhambane aurait pu être écrite par Marcel Aymé ou Zola) et termine par un éloge de la femme mozambicaine, ce en quoi il a bien raison quand on compare leur pugnacité à ce qu'ont fait pères, frères et époux depuis 1964 .

Un autre grand saut aux antipodes en plein dans un écran de fumée crevé par un historien engagé qui se met en devoir de démolir la propagande de plusieurs gouvernements successifs à Canberra? Je n'ai pas les connaissances nécessaires pour dire si Clinton Fernandes, lobbyiste pro-timorien, se laisse emporter par la passion ou plutôt s'il traduit la réalité politique dans les sphères du pouvoir. Dans ces milieux-là, mieux vaut être cuirassé si on ne veut pas succomber sous les coups de Noam Chomsky ou de ses adversaires. Donc - son titre dit tout ${ }^{15}$-, c'est parce qu'elle a eu beaucoup à se faire pardonner que l'Australie est venue - contrainte et pleine de remords - au secours de Timor en 1999-2000, après des années de collusion avec Djakarta. J'en avais la prémonition. Le livre semble en apporter la confirmation. Sous réserve de recevoir la démonstration du contraire.

Ce que je sais, c'est qu'en matière de relations publiques, il ne faut jamais être timide dans l'émission de la fumée. Elle pollue un peu, certes, mais moins que l'horrible fiction répandue par les médias et les littérateurs les plus hostiles au régime familial en place depuis 1968 en Guinée équatoriale. Puisque Alberto

14 M.A. López, Mozambique a pedacitos, Managua, Asociación para el desarrollo cultural Camino, 2006, 117 p., ISBN : 99926-29-69-X.

15 C. Fernandes, Reluctant Saviour. Australia, Indonesia and the Independence of East Timor, Melbourne, Scribe Publications, 2004, 138 p., ISBN : 1-920769-28-5. 
Michelini ${ }^{16}$, représentant personnel du Président du Conseil italien pour l'Afrique, nous dit sans broncher que ce petit État est « un modèle africain de développement », à l'issue d'une longue interview du neveu de Papa Macías ${ }^{17}$, c'est que moi non plus je n'ai rien compris à la bonté des intentions des hommes politiques équato-guinéens. Comme les gens de l'Estado Novo portugais, du MPLA, du Frelimo et du PAIGC (Parti africain pour l'indépendance de la Guinée et du Cap-Vert) m'ont déjà accusé de cette même incapacité à les comprendre, je bats ma coulpe, honteux et confus, et je m'arrête là pour ne pas aggraver mon cas davantage.

8 juin 2007, René Pélissier, CNRS

16 A. Michelini, Por una Guinea mejor. Guinea Ecuatorial: un modelo africano de desarrollo. Entrevista al Presidente de Guinea Ecuatorial T. Obiang Nguema Mbasogo, Chiasso (Suisse), Elvetica Edizioni, 2006, 102 p., photos couleur et noir et blanc, ISBN : 88-86639-46-5.

17 Cf. R. PÉLissier, Don Quichotte en Afrique. Voyages à la fin de l'Empire espagnol, 1992, 176 p. ; Spanish Africa - Afrique espagnole. Études sur la fin d'un Empire, 1957-1976, 2005, 224 p. ; AngolaGuinées-Mozambique-Sahara-Timor, etc. Une bibliographie internationale critique, 1990-2005, 2006, 748 p. [Orgeval (France), Éditions Pélissier pour ces ouvrages], trois livres curieusement introuvables dans les richissimes bibliothèques africanistes de Florence, Rome et Malabo. 


\section{Add dimension to your \\ sociology research --

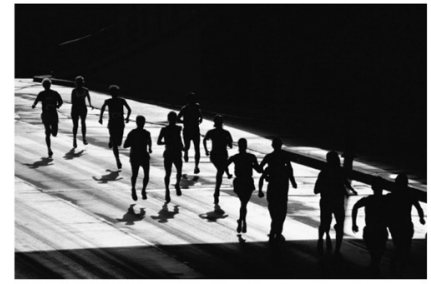 \\ ESA Sociological Abstracts}

Comprehensive, cost-effective, timely coverage of current ideas in sociological research

Abstracts of articles, books, and conference papers from nearly 2,000 journals published in 35 countries; citations of relevant dissertations as well as books and other media.

Now featuring:

- Cited references

- Backfiles to 1952

- Scholarprofiles

Now available with ProQuest Full-Text!

Contact sales@csa.com for trial Internet access or a sample issue

\section{ESA Sociological Ahstracts Discovery Prize}

Promoting teaching and learning in the social sciences through the use of CSA Sociological Abstracts

Entry information and past winners: info.csa.com/sociologicaldiscovery

\section{ProGuest CSA}

www.csa.com 